\title{
A Network Meta-Analysis on Randomized Trials Focusing on the Preventive Effect of Statins on Contrast-Induced Nephropathy
}

\author{
Mariangela Peruzzi, ${ }^{1}$ Leonardo De Luca, ${ }^{2}$ Henrik S. Thomsen, ${ }^{3}$ \\ Enrico Romagnoli, ${ }^{4}$ Fabrizio D'Ascenzo, ${ }^{5}$ Massimo Mancone, ${ }^{6}$ Gennaro Sardella, ${ }^{6}$ \\ Luigi Lucisano, ${ }^{6}$ Antonio Abbate, ${ }^{7}$ Giacomo Frati, ${ }^{1,8}$ and Giuseppe Biondi-Zoccai ${ }^{1,7}$ \\ ${ }^{1}$ Department of Medico-Surgical Sciences and Biotechnologies, Sapienza University of Rome, Corso della Repubblica 79, \\ 04100 Latina, Italy \\ ${ }^{2}$ Department of Cardiovascular Sciences, European Hospital, 00149 Rome, Italy \\ ${ }^{3}$ Department of Radiology, Copenhagen University Hospital, 2100 Herlev, Denmark \\ ${ }^{4}$ Division of Cardiology, Policlinico Casilino, 00169 Rome, Italy \\ ${ }^{5}$ Division of Cardiology, University of Turin, 10126 Turin, Italy \\ ${ }^{6}$ Department of Cardiovascular, Respiratory, Nephrologic and Geriatric Sciences, Sapienza University of Rome, 00185 Rome, Italy \\ ${ }^{7}$ VCU Pauley Heart Center, Virginia Commonwealth University, Richmond, VA 23298, USA \\ ${ }^{8}$ Department of Angiocardioneurology, IRCCS Neuromed, 86077 Pozzilli, Italy
}

Correspondence should be addressed to Mariangela Peruzzi; mariangela.peruzzi@uniromal.it

Received 16 April 2014; Accepted 6 August 2014; Published 7 September 2014

Academic Editor: Emmanuel A. Burdmann

Copyright ( 2014 Mariangela Peruzzi et al. This is an open access article distributed under the Creative Commons Attribution License, which permits unrestricted use, distribution, and reproduction in any medium, provided the original work is properly cited.

Contrast-induced nephropathy is a common complication of iodinated contrast administration. Statins may reduce the risk of contrast-induced nephropathy, but data remain inconclusive. We summarized the evidence based on statins for the prevention of contrast-induced nephropathy with a network meta-analysis. Randomized trials focusing on statins were searched and pooled with random-effect odds ratios. A total of 14 trials (6,160 patients) were included, focusing on atorvastatin (high/low dose), rosuvastatin (high dose), simvastatin (high/low dose), and placebo or no statin therapy before contrast administration. The risk of contrast-induced nephropathy was reduced by atorvastatin high dose and rosuvastatin high dose, with no difference between these two agents. Results for atorvastatin low dose and simvastatin (high/low dose) in comparison to placebo were inconclusive. Atorvastatin and rosuvastatin administered at high doses and before iodinated contrast administration have a consistent and beneficial preventive effect on contrast-induced nephropathy and may actually halve its incidence.

\section{Introduction}

Iodinated contrast-induced nephropathy (CIN) is an important cause of hospital-acquired acute renal injury [1]. Patients with acute coronary syndrome (ACS) and/or baseline renal dysfunction, as well as those undergoing percutaneous coronary intervention (PCI), are at particularly high risk of CIN, which, when occurring, may be followed by persistent worsening of renal function [2, 3]. To date, there have been many studies focusing on the pathophysiology, epidemiology, risk prognostication, and prevention of CIN. Yet, the pathophysiology of CIN remains unclear but may be related to direct renal tubular toxicity, vasoconstriction, and high oxidative stress $[4,5]$.

Statins are primarily used in cardiovascular medicine for their lipid-lowering effects. However, they have recently been shown to possess remarkable pleiotropic effects such as improving endothelial function as well as decreasing oxidative stress and inflammation [6]. In the context of cardiovascular disease, nitric oxide-derived oxidant species that promote atherogenesis are suppressed by statins [7]. 
Therefore, statins are considered as promising candidate agents for the prevention of CIN.

A number of studies have shown that statins may have a protective effect on CIN, but there remains uncertainty regarding time and way of administration of statins and it is not clear whether a specific statin is better than the others. Pairwise meta-analyses combine the results of homogeneous studies conducted on the same topic, whereas network metaanalyses evaluate simultaneously both direct and indirect comparisons across trials sharing one or more common comparators [21-23]. The purpose of our work was thus to perform a systematic review including both pairwise and network meta-analysis in order to evaluate more accurately the effect of statins compared to placebo or standard therapies for the prevention of CIN.

\section{Methods}

2.1. Design. The present review was conducted in keeping with the current guidelines from the Preferred Reporting Items for Systematic reviews and Meta-Analyses (PRISMA) group and from the Cochrane Collaboration. All reviewing activities were conducted by two independent reviewers (MP, GBZ), with divergences solved after consensus.

2.2. Search. MEDLINE/PubMed was searched for suitable studies according to Biondi-Zoccai et al., with the following substring: [24] (statin* OR atorvastatin OR rosuvastatin OR simvastatin OR lovastatin OR fluvastatin) AND (contrast AND (nephropathy OR ((renal OR kidney) AND (injury OR damage)))). In addition, the Cochrane Library, Google Scholar, and Scopus were searched for additional studies. No language restriction was enforced in order to minimize the risk of publication bias, actually leading to the inclusion of a study published only in Chinese [8]. Searches were last updated on November 14, 2013, with the exception of the MEDLINE/PubMed query, which was last performed on July $15,2014$.

2.3. Selection. Citations were initially screened at the title/abstract level. If potentially pertinent, they were then retrieved as full texts. Studies were included if randomized, allocating one or more groups to statin therapy and reporting dichotomous outcomes relevant to the assessment of CIN. Accordingly, studies were excluded if not randomized, duplicated, focusing on nonstatin strategies, or lacking suitable outcome details.

2.4. Abstraction and Validity. Study, patient, and procedural features were systematically extracted, with a particular focus on treatment strategy, type and volume of contrast, and CINrelated outcomes. The primary endpoint was the occurrence, at the longest in-hospital follow-up, of CIN, defined as a relative increase in serum creatinine $\geq 25 \%$ or an absolute increase in serum creatinine $\geq 0.5 \mathrm{mg} / \mathrm{dL}(44 \mu \mathrm{mol} / \mathrm{L})$, typically $48-72$ hours after the index procedure. In addition, the following endpoints were also collected at the longest in-hospital follow-up: absolute increase in serum creatinine $\geq 0.5 \mathrm{mg} / \mathrm{dL}$
( $44 \mu \mathrm{mol} / \mathrm{L})$; relative increase in serum creatinine $\geq 25 \%$. Conversely, the longest available clinical follow-up (thus well beyond hospitalization, when available) was exploited to abstract data on all-cause death and need for dialysis or renal replacement therapy. Study validity was appraised in keeping with ongoing recommendations distinguishing several sources of bias typical of randomized trials [23].

2.5. Analysis. Categorical variables are described as $n(\%)$ and continuous variables as median. Pairwise meta-analysis was performed within a frequentist framework, computing odds ratios (OR, 95\% confidence intervals) by means of a MantelHaenszel random-effect method [21]. Network meta-analysis was performed within a Bayesian framework computing odds ratios (95\% credible intervals) and probability of being best of any given treatment (Pbest) with a fixed-effect hierarchical model, sampling posterior probabilities by means of Markov chain Monte Carlo (MCMC) methods with Gibbs sampling from 150,000 iterations obtained after a 50,000-iteration training phase $[22,23]$. Convergence was appraised graphically according to Gelman and Rubin, and noninformative priors were used throughout. A fixed-effect model was used for network meta-analysis based on the star-shaped evidence network, which is typically analyzed with such an approach, and after having confirmed that model fit was not different from a random-effect and a fixed-effect model computing values for deviance information criterion (DIC). Pairwise heterogeneity was appraised with chi-squared tests, whereas network inconsistency was evaluated comparing the results stemming from consistent and inconsistent models [21, 23]. Small study effects (e.g., publication bias) were explored by visual inspection of funnel plots. Computations were performed with RevMan and WinBUGS [23].

\section{Results}

From an initial set of 33,030 citations retrieved from multiple databases, a total of 30 articles were analyzed as full reports according to our explicit selection criteria, finally yielding 14 studies eligible for inclusion in our review (Figure 1). These trials included a total of 6,160 patients, according to the following evidence network (Figures 2 and 3; Tables 1, 2, 3, and 4): 4 studies (706 subjects) compared atorvastatin high dose with atorvastatin low dose $[8,10,14,20], 5$ studies (1246 subjects) compared atorvastatin high dose with placebo or standard therapy without statins [13,15-18], 1 study (192 subjects) compared atorvastatin high dose with rosuvastatin high dose [11], 2 studies (3541 subjects) compared rosuvastatin high dose with placebo or standard therapy without statins $[4,12], 1$ study (247 subjects) compared simvastatin high dose with placebo or standard therapy without statins [9], and 1 study (228 subjects) compared simvastatin high dose with simvastatin low dose [19].

Studies were clinically diverse, being conducted in Asia and Europe, and included patients with diabetes mellitus (median prevalence 23\%) or mild to moderate chronic kidney disease (median baseline serum creatinine $1.0 \mathrm{mg} / \mathrm{dL} 1$ $(88 \mu \mathrm{mol} / \mathrm{L}))$. Several types of iodinated contrast media were 


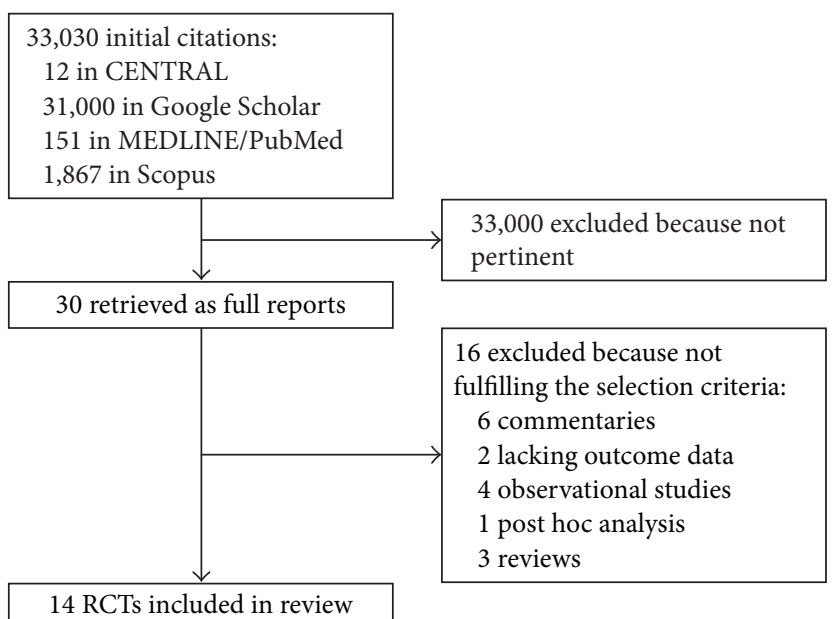

FIGURE 1: Review profile.

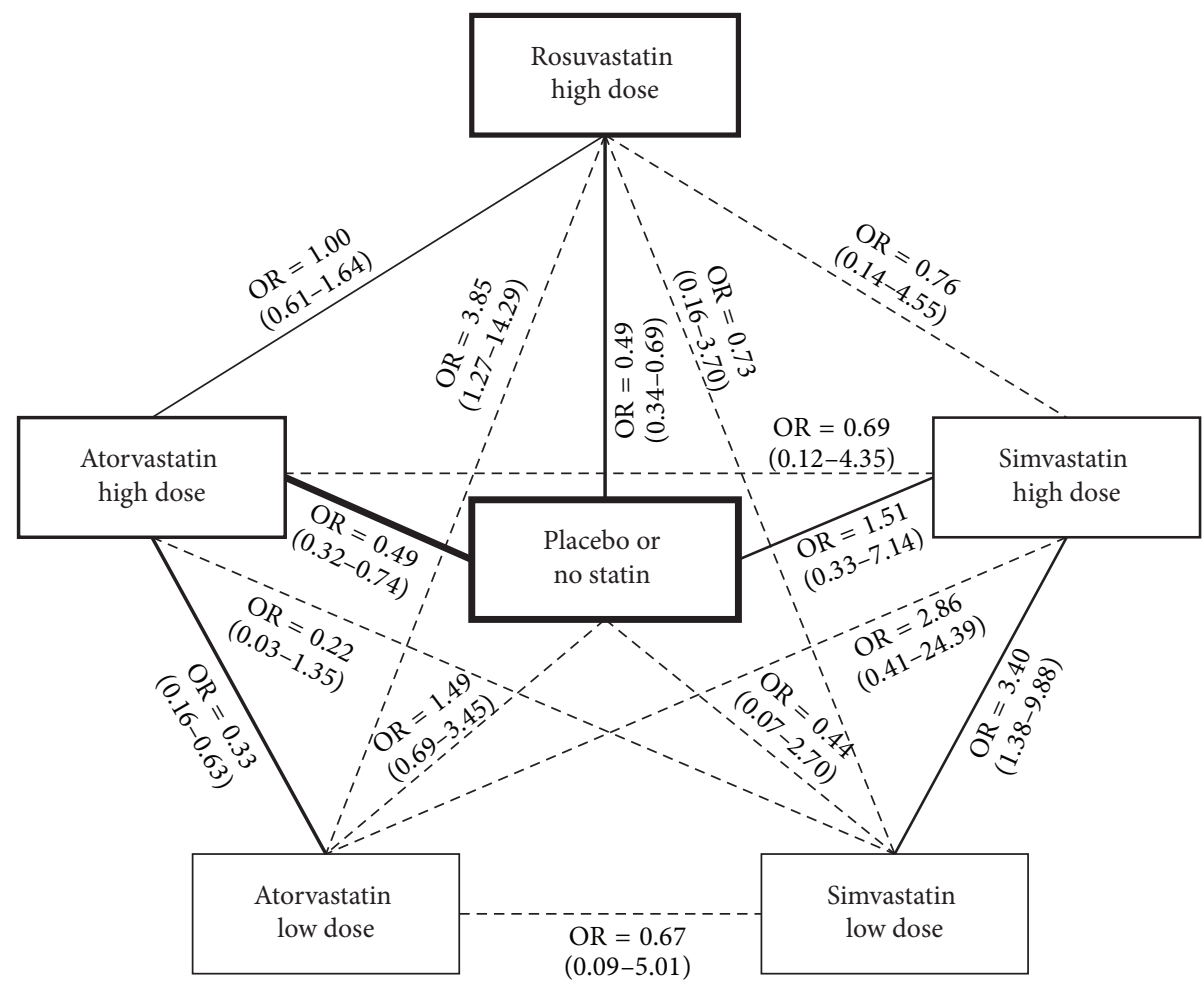

FIGURE 2: Evidence network. Continuous lines represent head-to-head randomized comparisons (the thickness of the line corresponding to the number of trials) with both direct and indirect effect estimates, whereas dashed lines represent only indirect effect estimates. The thickness of the rectangles corresponds to the patients receiving a specific treatment.

used, including iobitridol, iodixanol, iohexol, iopamidol, and iopromide (median volume $161 \mathrm{~mL}$ ). Study quality was adequate in most cases, but satisfactory details on randomization procedures and thorough double blinding were present only for 5 trials $[9,13,16-18]$.

Pairwise meta-analysis for CIN was first conducted to explore for overall clinical effects (online Figures 1-13 in Supplementary Material available online at http://dx.doi.org/10.1155/2014/213239). These computations showed that statins at high dose were associated with a reduced risk of $\mathrm{CIN}$ ( $\mathrm{OR}=0.47$ ( $95 \%$ confidence interval $0.37-0.60), P$ for effect $<0.001, P$ for heterogeneity $=0.59$, and I-squared $=0 \%)$, of an absolute increase in serum creatinine $\geq 0.5 \mathrm{mg} / \mathrm{dL}(\mathrm{OR}=0.57(0.32-1.02), P$ for effect $=0.06, P$ for heterogeneity $=0.26$, and I-squared $=25 \%)$, and of a relative increase in serum creatinine $\geq 25 \%$ ( OR $=0.35(0.17-0.69), P$ 


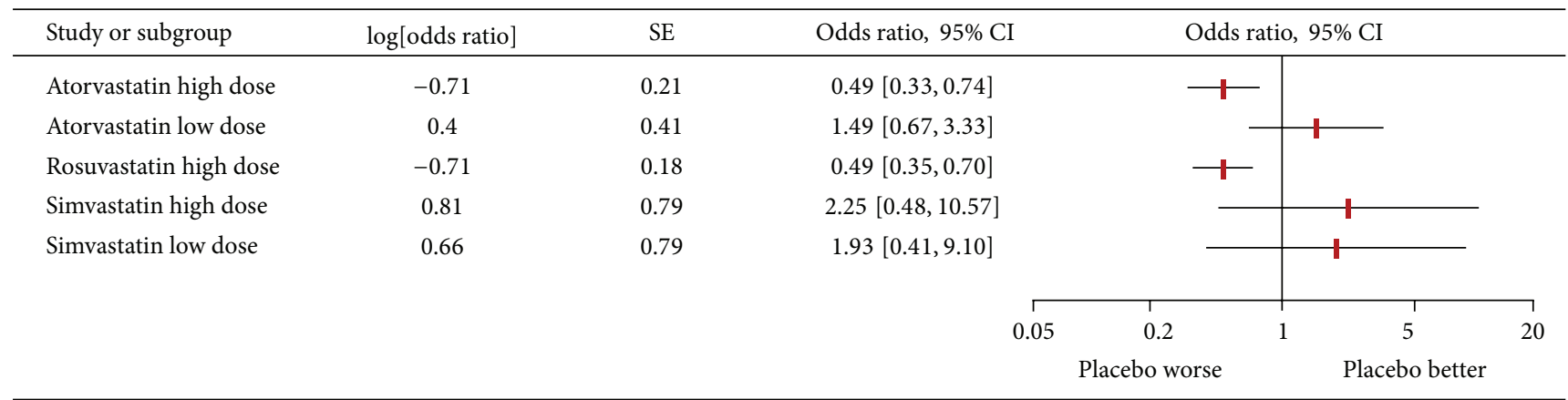

FIGURE 3: Forest plot summarizing risk estimates stemming from network meta-analysis comparing different statin regimens for the risk of contrast-induced nephropathy. $\mathrm{OR}=$ odds ratio; $\mathrm{CI}=$ confidence interval; $\mathrm{SE}=$ standard error.

TABLE 1: Included studies.

\begin{tabular}{|c|c|c|c|c|c|c|c|}
\hline First author & Year & Journal & Patients & $\begin{array}{c}\text { Multicenter } \\
\text { setting }\end{array}$ & Location & $\begin{array}{c}\text { Follow-up } \\
\text { (days) }\end{array}$ & Selection criteria \\
\hline Cao $[8]$ & 2012 & $\begin{array}{l}\text { Nan Fang Yi Ke Da } \\
\text { Xue Xue Bao }\end{array}$ & 180 & No & China & 3 & $\begin{array}{l}\text { ACS undergoing coronary } \\
\text { angiography or PCI without renal } \\
\text { failure }\end{array}$ \\
\hline Han [4] & 2013 & $\begin{array}{c}\text { Journal of the } \\
\text { American College of } \\
\text { Cardiology }\end{array}$ & 2998 & Yes & China & 30 & Type 2 DM and stage 2-3 CKD \\
\hline Jo [9] & 2008 & $\begin{array}{c}\text { American Heart } \\
\text { Journal }\end{array}$ & 247 & Yes & Korea & 180 & $\begin{array}{l}\text { CKD, statin naive, undergoing } \\
\text { coronary angiography }\end{array}$ \\
\hline Jo $[10]$ & 2014 & $\begin{array}{c}\text { Journal of } \\
\text { Cardiovascular } \\
\text { Medicine } \\
\end{array}$ & 218 & Yes & Korea & 180 & $\begin{array}{l}\text { STEMI undergoing emergency PCI } \\
\text { irrespective of renal dysfunction }\end{array}$ \\
\hline Kaya [11] & 2013 & Acta Cardiologica & 192 & No & Turkey & 2 & $\begin{array}{l}\text { STEMI undergoing emergency PCI } \\
\text { without renal dysfunction }\end{array}$ \\
\hline Leoncini $[12]$ & 2014 & $\begin{array}{c}\text { Journal of the } \\
\text { American College of } \\
\text { Cardiology }\end{array}$ & 543 & No & Italy & 180 & $\begin{array}{l}\text { NSTE-ACS, statin naive, selected for } \\
\text { early invasive strategy, without acute } \\
\text { or end-stage renal failure }\end{array}$ \\
\hline $\operatorname{Li}[13]$ & 2012 & Cardiology & 161 & No & China & 30 & $\begin{array}{l}\text { STEMI undergoing emergency PCI } \\
\text { without renal dysfunction }\end{array}$ \\
\hline $\operatorname{Li}[14]$ & 2014 & $\begin{array}{l}\text { Scientific World } \\
\text { Journal }\end{array}$ & 208 & No & China & 1 & $\begin{array}{l}\text { Patients undergoing coronary } \\
\text { angiography or angioplasty }\end{array}$ \\
\hline Özhan [15] & 2010 & Angiology & 130 & No & Turkey & 2 & $\begin{array}{l}\text { Patients undergoing coronary } \\
\text { angiography without renal } \\
\text { insufficiency (serum creatinine } \\
<1.5 \mathrm{mg} / \mathrm{dL} \text { ) }\end{array}$ \\
\hline Patti [16] & 2011 & $\begin{array}{l}\text { American Journal of } \\
\text { Cardiology }\end{array}$ & 241 & Yes & Italy & 2 & $\begin{array}{l}\text { NSTE-ACS, statin naive, receiving } \\
\text { early PCI }\end{array}$ \\
\hline Quintavalle [17] & 2012 & Circulation & 410 & Yes & Italy & 365 & $\begin{array}{l}\text { CKD, statin naive, scheduled for } \\
\text { elective coronary angiography or PCI }\end{array}$ \\
\hline Toso $[18]$ & 2010 & $\begin{array}{l}\text { American Journal of } \\
\text { Cardiology }\end{array}$ & 304 & No & Italy & 30 & $\begin{array}{l}\mathrm{CKD} \text {, statin naive, without end-stage } \\
\text { renal failure requiring dialysis }\end{array}$ \\
\hline Xinwei [19] & 2009 & $\begin{array}{c}\text { American Journal of } \\
\text { Cardiology }\end{array}$ & 228 & No & China & 7 & ACS undergoing PCI \\
\hline Zhou [20] & 2009 & $\begin{array}{l}\text { Zhonghua Xin Xue } \\
\text { Guan Bing Za Zhi }\end{array}$ & 100 & No & China & 3 & Coronary angiography or PCI \\
\hline
\end{tabular}

ACS = acute coronary syndrome; $\mathrm{CKD}$ = chronic kidney disease; $\mathrm{DM}$ = diabetes mellitus; NSTE-ACS = non-ST-elevation ACS; PCI = percutaneous coronary intervention; STEMI = ST-elevation myocardial infarction. 
TABLE 2: Patient and procedural features.

\begin{tabular}{|c|c|c|c|c|c|c|c|c|}
\hline First author & Year & Experimental therapy & $\begin{array}{l}\text { Control } \\
\text { therapy }\end{array}$ & $\begin{array}{l}\text { Contrast } \\
\text { type }\end{array}$ & $\begin{array}{l}\text { Median } \\
\text { contrast } \\
\text { volume }\end{array}$ & $\begin{array}{l}\text { Age } \\
\text { (years) }\end{array}$ & $\begin{array}{l}\text { Diabetes } \\
\text { mellitus }\end{array}$ & $\begin{array}{l}\text { Baseline } \\
\text { serum } \\
\text { creatinine } \\
(\mathrm{mg} / \mathrm{dL})\end{array}$ \\
\hline Cao $[8]$ & 2012 & $\begin{array}{l}\text { Atorvastatin } 40 \mathrm{mg} / \text { day started } 3 \\
\text { days before angioplasty followed } \\
\text { by atorvastatin } 20 \mathrm{mg} / \text { day }\end{array}$ & $\begin{array}{l}\text { Atorvastatin } \\
20 \mathrm{mg} / \text { day }\end{array}$ & NA & 161 & 63 & $20 \%$ & NA \\
\hline Han [4] & 2013 & $\begin{array}{l}\text { Rosuvastatin } 10 \mathrm{mg} / \text { day from } 2 \\
\text { days before to } 3 \text { days after } \\
\text { contrast }\end{array}$ & No statin & Iodixanol & 115 & 61 & $100 \%$ & 1.1 \\
\hline Jo [9] & 2008 & $\begin{array}{l}\text { Simvastatin } 40+40 \mathrm{mg} \text { before } \\
\text { angiography followed by } \\
\text { simvastatin } 40+40 \mathrm{mg} \\
\text { afterwards }\end{array}$ & Placebo & Iodixanol & 182 & 65 & $26 \%$ & 1.2 \\
\hline Jo [10] & 2014 & $\begin{array}{l}\text { Atorvastatin } 80 \mathrm{mg} \text { before } \\
\text { angiography followed by } \\
\text { atorvastatin } 80 \mathrm{mg} / \text { day for } 5 \text { days } \\
\text { and then } 10 \mathrm{mg} / \text { day }\end{array}$ & $\begin{array}{l}\text { Atorvastatin } \\
10 \mathrm{mg} / \text { day }\end{array}$ & NA & NA & 60 & $26 \%$ & 1.1 \\
\hline Kaya [11] & 2013 & $\begin{array}{l}\text { Atorvastatin } 80 \mathrm{mg} \text { before } \\
\text { angiography }\end{array}$ & $\begin{array}{l}\text { Rosuvastatin } \\
40 \text { mg before } \\
\text { angiography }\end{array}$ & Iopromide & 153 & 63 & $20 \%$ & 0.9 \\
\hline Leoncini [12] & 2014 & $\begin{array}{l}\text { Rosuvastatin } 40 \mathrm{mg} \text { at admission } \\
\text { followed by } 20 \mathrm{mg} / \text { day }\end{array}$ & No statin & Iodixanol & 261 & 66 & $21 \%$ & 1.0 \\
\hline $\mathrm{Li}[13]$ & 2012 & $\begin{array}{l}\text { Atorvastatin } 80 \mathrm{mg} \text { at admission } \\
\text { followed by atorvastatin } 40 \mathrm{mg} \\
\text { after angiography }\end{array}$ & Placebo & Iopromide & 102 & 66 & $28 \%$ & 0.9 \\
\hline Li [14] & 2014 & $\begin{array}{l}\text { Atorvastatin } 40 \mathrm{mg} \text { before } \\
\text { angiography, followed by } \\
\text { atorvastatin } 40 \mathrm{mg} / \text { day }\end{array}$ & $\begin{array}{l}\text { Atorvastatin } \\
20 \mathrm{mg} / \text { day }\end{array}$ & Iopamidol & 186 & 61 & $24 \%$ & 0.9 \\
\hline Özhan [15] & 2010 & $\begin{array}{l}\text { Atorvastatin } 80 \mathrm{mg} \text { before } \\
\text { angiography, followed by } \\
\text { atorvastatin } 80 \mathrm{mg} 48 \text { hours after } \\
\text { contrast administration }\end{array}$ & No statin & Iopamidol & 95 & 55 & $16 \%$ & 0.8 \\
\hline Patti [16] & 2011 & $\begin{array}{l}\text { Atorvastatin } 80 \mathrm{mg} 12 \mathrm{hrs} \text { before } \\
\text { angiography; further } 40 \mathrm{mg} \\
\text { preprocedure ( } 2 \text { hrs before), } \\
\text { followed by atorvastatin } \\
40 \mathrm{mg} / \text { day }\end{array}$ & Placebo & Iobitridol & 211 & 66 & $27 \%$ & 1.0 \\
\hline Quintavalle [17] & 2012 & $\begin{array}{l}\text { Atorvastatin } 80 \mathrm{mg} \text { (within } \\
24 \text { hrs before contrast exposure), } \\
\text { followed by atorvastatin } \\
20 \mathrm{mg} / \text { day }\end{array}$ & No statin & Iodixanol & 180 & 70 & $41 \%$ & 1.3 \\
\hline Toso [18] & 2010 & $\begin{array}{l}\text { Atorvastatin } 80 \mathrm{mg} 48 \text { hours } \\
\text { before and } 48 \text { hours after } \\
\text { contrast administration }\end{array}$ & Placebo & Iodixanol & 157 & 75 & $21 \%$ & 1.2 \\
\hline Xinwei [19] & 2009 & $\begin{array}{l}\text { Simvastatin } 80 \mathrm{mg} \text { from } \\
\text { admission to day before PCI, } \\
\text { followed by simvastatin } \\
20 \mathrm{mg} / \text { day }\end{array}$ & $\begin{array}{l}\text { Simvastatin } \\
20 \mathrm{mg} / \text { day }\end{array}$ & $\begin{array}{l}\text { Iodixanol } \\
\text { or iohexol }\end{array}$ & 233 & 65 & $21 \%$ & 0.8 \\
\hline Zhou [20] & 2009 & $\begin{array}{l}\text { Atorvastatin } 80 \mathrm{mg} / \text { day before } \\
\text { the procedure, } 10 \mathrm{mg} / \text { day for } 6 \\
\text { days after procedure }\end{array}$ & $\begin{array}{l}\text { Atorvastatin } \\
10 \mathrm{mg} / \text { day for } \\
7 \text { days }\end{array}$ & Iopamidol & 116 & 61 & $20 \%$ & 1.1 \\
\hline
\end{tabular}

$\mathrm{NA}=$ not available or applicable . 
TABLE 3: Internal validity of included studies.

\begin{tabular}{|c|c|c|c|c|c|c|c|}
\hline First author & Year & $\begin{array}{l}\text { Inadequate allocation } \\
\text { sequence generation? }\end{array}$ & $\begin{array}{l}\text { Inadequate allocation } \\
\text { concealment? }\end{array}$ & $\begin{array}{l}\text { Inadequate } \\
\text { blinding? }\end{array}$ & $\begin{array}{c}\text { Incomplete } \\
\text { outcome } \\
\text { data? }\end{array}$ & $\begin{array}{l}\text { Selective outcome } \\
\text { reporting? }\end{array}$ & $\begin{array}{c}\text { Risk of } \\
\text { other bias? }\end{array}$ \\
\hline Cao [8] & 2012 & Unclear & Unclear & Unclear & No & $\begin{array}{c}\text { Yes (lack of some } \\
\text { individual CIN data) }\end{array}$ & No \\
\hline Han [4] & 2013 & No & No & Yes (open label) & No & $\begin{array}{c}\text { Yes (lack of some } \\
\text { individual CIN data) }\end{array}$ & No \\
\hline Jo [9] & 2008 & No & No & No & No & $\begin{array}{c}\text { Yes (lack of some } \\
\text { individual CIN data) }\end{array}$ & No \\
\hline Jo [10] & 2014 & No & Unclear & Yes (open label) & No & $\begin{array}{c}\text { Yes (lack of some } \\
\text { individual CIN data) }\end{array}$ & No \\
\hline Kaya [11] & 2013 & Unclear & Unclear & Yes (open label) & No & $\begin{array}{c}\text { Yes (lack of some } \\
\text { individual CIN data) }\end{array}$ & No \\
\hline Leoncini [12] & 2014 & No & Yes (open-label list) & Yes (open label) & No & $\begin{array}{c}\text { Yes (lack of some } \\
\text { individual CIN data) }\end{array}$ & No \\
\hline $\operatorname{Li}[13]$ & 2012 & No & No & No & No & $\begin{array}{c}\text { Yes (lack of some } \\
\text { individual CIN data) }\end{array}$ & No \\
\hline $\mathrm{Li}[14]$ & 2014 & Unclear & Unclear & Yes (open label) & No & $\begin{array}{c}\text { Yes (lack of some } \\
\text { individual CIN data) }\end{array}$ & No \\
\hline Özhan [15] & 2010 & Unclear & Unclear & Unclear & No & $\begin{array}{c}\text { Yes (lack of some } \\
\text { individual CIN data) }\end{array}$ & No \\
\hline Patti [16] & 2011 & No & No & No & No & $\begin{array}{c}\text { Yes (lack of some } \\
\text { individual CIN data) }\end{array}$ & No \\
\hline Quintavalle [17] & 2012 & No & No & No & No & $\begin{array}{c}\text { Yes (lack of some } \\
\text { individual CIN data) }\end{array}$ & No \\
\hline Toso $[18]$ & 2010 & No & No & No & No & $\begin{array}{c}\text { Yes (lack of some } \\
\text { individual CIN data) }\end{array}$ & No \\
\hline Xinwei [19] & 2009 & No & Yes (open-label study) & Yes (open label) & No & $\begin{array}{c}\text { Yes (lack of some } \\
\text { individual CIN data) }\end{array}$ & No \\
\hline Zhou [20] & 2009 & Unclear & Unclear & Unclear & No & $\begin{array}{c}\text { Yes (lack of some } \\
\text { individual CIN data) }\end{array}$ & No \\
\hline
\end{tabular}

$\mathrm{CIN}=$ contrast-induced nephropathy.

for effect $=0.003, P$ for heterogeneity $=0.57$, and I-squared $=0 \%)$. In addition, statins at high dose were associated with trends toward reduced risks of in-hospital dialysis $(\mathrm{OR}=$ 0.27 (0.07-1.09), $P$ for effect $=0.07, P$ for heterogeneity $=$ 1.0 , and I-squared $=0 \%)$ and death at a median follow-up of 3 months $(\mathrm{OR}=0.80(0.31-2.10), P$ for effect $=0.65, P$ for heterogeneity $=0.61$, and I-squared $=0 \%)$.

Subsequently, network meta-analysis was performed for the risk of CIN, distinguishing atorvastatin high dose, atorvastatin low dose, rosuvastatin high dose, simvastatin high dose, and simvastatin low dose. These computations showed that atorvastatin high dose was the regimen which was most likely to be beneficial (OR versus placebo $=0.49$ ( $95 \%$ credible interval $0.32-0.74)$, Pbest $=34 \%)$, followed by rosuvastatin high dose (OR versus placebo $=0.49(0.34-0.69)$, Pbest $=$ $34 \%)$. Head-to-head comparison of atorvastatin high dose versus rosuvastatin high dose suggested a very similar effect $(\mathrm{OR}=1.00(0.61-1.64))$. Data for simvastatin high dose were apparently favorable, but inconclusive $(\mathrm{OR}$ versus placebo $=$ $0.66(0.14-3.04)$, Pbest $=32 \%)$, whereas data for atorvastatin low dose and simvastatin low dose were clearly not in favor of clinical use to prevent CIN (resp. OR versus placebo = $1.49(0.69-3.45)$, Pbest $<0.1 \%$, and OR versus placebo $=2.25$
(0.37-14.61), Pbest $=0.2 \%)$. Additional network analyses for the other CIN endpoints, dialysis, or death were not possible for the paucity of reported events.

Consistency of pairwise and network analyses was satisfactory, in light of the low I-squared estimates and the similar results yielded by consistent, inconsistent, fixed-effect, and random-effect Bayesian models. Even sensitivity analysis excluding the largest study (Han et al.) [4] confirmed in direction and magnitude of effects the overall analysis (Table 5). Finally, no clear evidence of small study effects was found at funnel plot inspection (online Figures 3, 6, 9, 11, and 13).

\section{Discussion}

This review, the first in the literature exploiting the totality of the evidence base with the novel mixed treatment comparison approach for comparative effectiveness analysis, confirms that statins at high dose reduce the risk of CIN in patients undergoing coronary angiography or revascularization with current iodinated contrast media, with potentially beneficial effects also on the risk of in-hospital dialysis. Moreover, 
TABLE 4: Effect estimates for the risk of contrast-induced nephropathy. Results are reported as probability of being treatment (Pbest) and odds ratios (OR) with $95 \%$ credible intervals of a given row item versus a corresponding column item. Thus, OR $<1$ indicates lower risk in the corresponding row item and $\mathrm{OR}>1$ indicates lower risk in the corresponding column item.

\begin{tabular}{|c|c|c|c|c|c|c|}
\hline Treatment & $\begin{array}{l}\text { Atorvastatin } \\
\text { high dose }\end{array}$ & $\begin{array}{c}\text { Atorvastatin low } \\
\text { dose }\end{array}$ & $\begin{array}{l}\text { Rosuvastatin } \\
\text { high dose }\end{array}$ & $\begin{array}{l}\text { Simvastatin } \\
\text { high dose }\end{array}$ & $\begin{array}{l}\text { Simvastatin low } \\
\text { dose }\end{array}$ & $\begin{array}{c}\text { Placebo or no } \\
\text { statin }\end{array}$ \\
\hline $\begin{array}{l}\text { Atorvastatin } \\
\text { high dose }\end{array}$ & Pbest $=34 \%$ & $\begin{array}{c}\mathrm{OR}=0.33 \\
(0.16-0.63)\end{array}$ & $\begin{array}{l}\mathrm{OR}=1.00 \\
(0.61-1.64)\end{array}$ & $\begin{array}{l}\mathrm{OR}=0.69 \\
(0.12-4.35)\end{array}$ & $\begin{array}{l}\mathrm{OR}=0.22 \\
(0.03-1.35)\end{array}$ & $\begin{array}{c}\mathrm{OR}=0.49 \\
(0.32-0.74)\end{array}$ \\
\hline $\begin{array}{l}\text { Atorvastatin low } \\
\text { dose }\end{array}$ & $\begin{array}{c}\mathrm{OR}=3.05 \\
(1.59-6.21)\end{array}$ & Pbest $<0.1 \%$ & $\begin{array}{c}\mathrm{OR}=3.85 \\
(1.27-14.29)\end{array}$ & $\begin{array}{c}\mathrm{OR}=2.86 \\
(0.41-24.39)\end{array}$ & $\begin{array}{l}\mathrm{OR}=0.67 \\
(0.09-5.01)\end{array}$ & $\begin{array}{c}\mathrm{OR}=1.49 \\
(0.69-3.45)\end{array}$ \\
\hline $\begin{array}{l}\text { Rosuvastatin } \\
\text { high dose }\end{array}$ & $\begin{array}{l}\mathrm{OR}=1.00 \\
(0.61-1.63)\end{array}$ & $\begin{array}{c}\mathrm{OR}=0.26 \\
(0.07-0.79)\end{array}$ & Pbest $=34 \%$ & $\begin{array}{c}\mathrm{OR}=0.76 \\
(0.14-4.55)\end{array}$ & $\begin{array}{l}\mathrm{OR}=0.73 \\
(0.16-3.70)\end{array}$ & $\begin{array}{c}\mathrm{OR}=0.49 \\
(0.34-0.69)\end{array}$ \\
\hline $\begin{array}{l}\text { Simvastatin } \\
\text { high dose }\end{array}$ & $\begin{array}{l}\mathrm{OR}=1.44 \\
(0.23-8.11)\end{array}$ & $\begin{array}{c}\mathrm{OR}=0.35 \\
(0.04-2.42)\end{array}$ & $\begin{array}{l}\mathrm{OR}=1.32 \\
(0.22-7.13)\end{array}$ & Pbest $=32 \%$ & $\begin{array}{c}\mathrm{OR}=0.29 \\
(0.10-0.72)\end{array}$ & $\begin{array}{c}\mathrm{OR}=0.66 \\
(0.14-3.04)\end{array}$ \\
\hline $\begin{array}{l}\text { Simvastatin low } \\
\text { dose }\end{array}$ & $\begin{array}{c}\mathrm{OR}=4.61 \\
(0.74-30.37)\end{array}$ & $\begin{array}{c}\mathrm{OR}=1.50 \\
(0.20-10.86)\end{array}$ & $\begin{array}{c}\mathrm{OR}=1.37 \\
(0.27-6.42)\end{array}$ & $\begin{array}{c}\mathrm{OR}=3.40 \\
(1.38-9.88)\end{array}$ & Pbest $=0.2 \%$ & $\begin{array}{c}\mathrm{OR}=2.25 \\
(0.37-14.61)\end{array}$ \\
\hline $\begin{array}{l}\text { Placebo or } \\
\text { no statin }\end{array}$ & $\begin{array}{c}\mathrm{OR}=2.05 \\
(1.35-3.09)\end{array}$ & $\begin{array}{c}\mathrm{OR}=0.67 \\
(0.29-1.44)\end{array}$ & $\begin{array}{c}\mathrm{OR}=2.04 \\
(1.44-2.94)\end{array}$ & $\begin{array}{c}\mathrm{OR}=1.51 \\
(0.33-7.14)\end{array}$ & $\begin{array}{l}\mathrm{OR}=0.44 \\
(0.07-2.70)\end{array}$ & Pbest $=0$ \\
\hline
\end{tabular}

TABLE 5: Effect estimates for the risk of contrast-induced nephropathy excluding the study by Han et al. [4]. Results are reported as probability of being best treatment (Pbest) and odds ratios (OR) with 95\% credible intervals of a given row item versus a corresponding column item. Thus, $\mathrm{OR}<1$ indicates lower risk in the corresponding row item and OR $>1$ indicates lower risk in the corresponding column item.

\begin{tabular}{|c|c|c|c|c|c|c|}
\hline Treatment & $\begin{array}{l}\text { Atorvastatin } \\
\text { high dose }\end{array}$ & $\begin{array}{c}\text { Atorvastatin low } \\
\text { dose }\end{array}$ & $\begin{array}{l}\text { Rosuvastatin } \\
\text { high dose }\end{array}$ & $\begin{array}{l}\text { Simvastatin } \\
\text { high dose }\end{array}$ & $\begin{array}{c}\text { Simvastatin low } \\
\text { dose }\end{array}$ & $\begin{array}{c}\text { Placebo or no } \\
\text { statin }\end{array}$ \\
\hline $\begin{array}{l}\text { Atorvastatin } \\
\text { high dose }\end{array}$ & Pbest $=20 \%$ & $\begin{array}{c}\mathrm{OR}=0.32 \\
(0.15-0.65)\end{array}$ & $\begin{array}{c}\mathrm{OR}=1.22 \\
(0.66-2.33)\end{array}$ & $\begin{array}{l}\mathrm{OR}=0.65 \\
(0.15-3.85)\end{array}$ & $\begin{array}{l}\mathrm{OR}=0.19 \\
(0.03-1.37)\end{array}$ & $\begin{array}{c}\mathrm{OR}=0.47 \\
(0.31-0.70)\end{array}$ \\
\hline $\begin{array}{l}\text { Atorvastatin low } \\
\text { dose }\end{array}$ & $\begin{array}{c}\mathrm{OR}=3.12 \\
(1.53-6.73)\end{array}$ & Pbest $<0.1 \%$ & $\begin{array}{c}\mathrm{OR}=3.85 \\
(1.47-11.09)\end{array}$ & $\begin{array}{c}\mathrm{OR}=2.08 \\
(0.36-14.08)\end{array}$ & $\begin{array}{l}\mathrm{OR}=0.60 \\
(0.08-4.98)\end{array}$ & $\begin{array}{l}\mathrm{OR}=1.45 \\
(0.62-3.45)\end{array}$ \\
\hline $\begin{array}{l}\text { Rosuvastatin } \\
\text { high dose }\end{array}$ & $\begin{array}{l}\mathrm{OR}=0.82 \\
(0.43-1.51)\end{array}$ & $\begin{array}{c}\mathrm{OR}=0.26 \\
(0.10-0.68)\end{array}$ & Pbest $=34 \%$ & $\begin{array}{c}\mathrm{OR}=0.53 \\
(0.12-3.23)\end{array}$ & $\begin{array}{l}\mathrm{OR}=0.16 \\
(0.02-1.22)\end{array}$ & $\begin{array}{c}\mathrm{OR}=0.38 \\
(0.22-0.65)\end{array}$ \\
\hline $\begin{array}{l}\text { Simvastatin } \\
\text { high dose }\end{array}$ & $\begin{array}{c}\mathrm{OR}=1.54 \\
(0.26-6.87)\end{array}$ & $\begin{array}{l}\mathrm{OR}=0.48 \\
(0.07-2.77)\end{array}$ & $\begin{array}{l}\mathrm{OR}=1.90 \\
(0.31-8.56)\end{array}$ & Pbest $=20 \%$ & $\begin{array}{l}\mathrm{OR}=0.29 \\
(0.10-0.78)\end{array}$ & $\begin{array}{l}\mathrm{OR}=0.72 \\
(0.13-3.06)\end{array}$ \\
\hline $\begin{array}{l}\text { Simvastatin low } \\
\text { dose }\end{array}$ & $\begin{array}{c}\mathrm{OR}=5.24 \\
(0.73-32.12)\end{array}$ & $\begin{array}{c}\mathrm{OR}=1.67 \\
(0.20-12.66)\end{array}$ & $\begin{array}{c}\mathrm{OR}=6.46 \\
(0.82-41.28)\end{array}$ & $\begin{array}{c}\mathrm{OR}=3.42 \\
(1.28-10.03)\end{array}$ & Pbest $=0.2 \%$ & $\begin{array}{c}\mathrm{OR}=2.46 \\
(0.35-14.17)\end{array}$ \\
\hline $\begin{array}{l}\text { Placebo or } \\
\text { no statin }\end{array}$ & $\begin{array}{c}\mathrm{OR}=2.15 \\
(1.42-3.28)\end{array}$ & $\begin{array}{l}\mathrm{OR}=0.69 \\
(0.29-1.61)\end{array}$ & $\begin{array}{c}\mathrm{OR}=2.63 \\
(1.54-4.55)\end{array}$ & $\begin{array}{c}\mathrm{OR}=1.39 \\
(0.33-7.69)\end{array}$ & $\begin{array}{c}\mathrm{OR}=0.41 \\
(0.07-2.86)\end{array}$ & Pbest $=0$ \\
\hline
\end{tabular}

network meta-analysis suggests that atorvastatin and rosuvastatin at high dose represent the first choice regimens in order to achieve a consistent and beneficial preventive effect on CIN and may actually halve its incidence.

Invasive angiography exploiting iodinated contrast media may be complicated in several cases by CIN, with a negative impact on prognosis, hospital stay, and costs [25]. Accordingly, means to prevent it may yield important and clinically relevant benefits. Yet, its pathophysiology remains unclear. It may be due to direct toxicity on the renal tubular epithelium, oxidative stress, ischemic injury, and renal tubular obstruction, as any change in nitric oxide, prostaglandins, endothelin, and adenosine in the vasoregulation pathway could contribute to worsen medullary ischemia [26]. Many preventive strategies and treatments have been proposed for CIN, although only few are with a demonstrated clinical efficacy $[25,27,28]$.

In addition to their impact on cholesterol, statins are known to have multiple nonlipid lowering (i.e., pleiotropic) effects, which include several mechanisms involving inflammation responses, endothelial function, oxidative stress, and apoptotic pathways [17]. Preventing contrast-induced renal cell apoptosis seems to play an important role in statins effects on CIN. Because inflammation is unquestionably involved in the pathogenesis of kidney injury, its modulation could be part of the mechanism expounding the reduced incidence of CIN after treatment with statins. The antioxidant effect of statins in addition to other antioxidant compounds (sodium bicarbonate solution and $\mathrm{N}$-acetylcysteine) seems to reduce the occurrence of CIN just through scavenging reactive oxygen species (ROS) [14]. Khanal et al. firstly reported, in a retrospective study that evaluated a large cohort of patients who underwent PCI, that patients on chronic statin therapy before the procedure had a significantly lower incidence of CIN [29]. Thereafter, several prospective nonrandomized or randomized placebo controlled trials suggested that a shortterm regimen of high-dose statins before and after contrast exposure, in addition to standard measures for preventing 
kidney deterioration, might decrease the incidence of CIN. Nevertheless, the type and the dose threshold of statins to reduce the risk of CIN remained uncertain.

Our meta-analysis represents the first work to exploit network meta-analytic methods to compare different statins in their CIN preventive effects. It shows that atorvastatin and rosuvastatin, when administered at high dose, represent the most effective preventive strategy. Accordingly, the present work provides important findings in support of the favorable risk-benefit balance of statins to prevent $\mathrm{CIN}$, as offering patients a short-term yet high-dose statin regimen before administration of iodinated contrast appears as a remarkably safe, simple, and effective intervention.

Focusing on current pharmacopeia, all statins are administered in active form except lovastatin and simvastatin which are given as a prodrug and converted in vivo into the liver. More of $90 \%$ of statins are bound to plasma proteins, but rosuvastatin has a lower rate of link $(88 \%)$. This is due to the hydrophilicity features of this molecule limiting the need for its transport in the blood through albumin. Hydrophilic statins encompass acute pleiotropic effects. Moreover, they do not undergo cytochrome P-450 3A4 metabolism in the liver. These features may explain, at least in part, potential differences between statins in CIN prevention. In addition, as CIN is a paradigm of mutual hypoxic and toxic renal parenchymal injury mediated, to large extent, by an increased production of mediators of inflammation and ROS and clinical as well as experimental findings clearly illustrate that hypoxia and enhanced ROS formation within the kidney following contrast media administration play a critical role in the development of CIN [30], we speculate that atorvastatin and rosuvastatin may be more effective than other statins probably due to their higher anti-inflammation and antioxidant features.

This work has several limitations, including those typical of systematic reviews, pairwise meta-analyses and mixed treatment comparisons [21, 23]. More poignantly to the current findings, we exploited an evidence network with a prevalent star shape. In addition, we relied on surrogate outcomes for renal function, which have been called into question for being overly sensitive and subject to substantial between- and within-individual variability. In particular, relative increases in serum creatinine $\geq 25 \%$ may occur in many patients irrespectively of the amount of administered contrast. In addition, no study focused on noncoronary contrast media administration, and thus the implications of our results for other imaging procedures (e.g., computed tomography) are open to individual interpretation. Finally, due to the lack of patient-level data, key moderators on the risk of CIN, such as means of contrast administration (intravenous versus intra-arterial), volume of contrast, type of contrast [25], and baseline use of statins, could not be appraised quantitatively.

\section{Conclusion}

Atorvastatin and rosuvastatin, when administered at high doses and before iodinated contrast administration, have a consistent and beneficial preventive effect on CIN and may actually halve its incidence.

\section{Conflict of Interests}

Drs. Biondi-Zoccai and De Luca have consulted and lectured for Astra Zeneca.

\section{Acknowledgment}

Dr. Biondi-Zoccai was, at the time of the preparation of this paper, the Congdon Visiting Scholar at the VCU Pauley Heart Center, Virginia Commonwealth University, Richmond, VA, USA.

\section{References}

[1] J. Golshahi, H. Nasri, and M. Gharipour, "Contrast-induced nephropathy; A literature review," Journal of Nephropathology, vol. 3, no. 2, pp. 51-56, 2014.

[2] T. Senoo, M. Motohiro, H. Kamihata et al., "Contrast-induced nephropathy in patients undergoing emergency percutaneous coronary intervention for acute coronary syndrome," The American Journal of Cardiology, vol. 105, no. 5, pp. 624-628, 2010.

[3] J. Wi, Y. G. Ko, J. S. Kim et al., "Impact of contrast-induced acute kidney injury with transient or persistent renal dysfunction on long-term outcomes of patients with acute myocardial infarction undergoing percutaneous coronary intervention," Heart, vol. 97, no. 21, pp. 1753-1757, 2011.

[4] Y. Han, G. Zhu, L. Han et al., "Short-term rosuvastatin therapy for prevention of contrast-induced acute kidney injury in patients with diabetes and chronic kidney disease," Journal of the American College of Cardiology, vol. 63, no. 1, pp. 62-70, 2013.

[5] S. N. Heyman, J. Reichman, and M. Brezis, "Pathophysiology of radiocontrast nephropathy: a role for medullary hypoxia," Investigative Radiology, vol. 34, no. 11, pp. 685-691, 1999.

[6] J. A. Farmer, "Pleiotropic effects of statins," Current Atherosclerosis Reports, vol. 2, no. 3, pp. 208-217, 2000.

[7] M. H. Shishehbor, R. J. Aviles, M. Brennan et al., "Association of nitrotyrosine levels with cardiovascular disease and modulation by statin therapy," The Journal of the American Medical Association, vol. 289, no. 13, pp. 1675-1680, 2003.

[8] S. Cao, P. Wang, K. Cui, L. Zhang, and Y. Hou, "Atorvastatin prevents contrast agent-induced renal injury in patients undergoing coronary angiography by inhibiting oxidative stress," Nan Fang Yi Ke Da Xue Xue Bao, vol. 32, no. 11, pp. 1600-1602, 2012.

[9] S. Jo, B. Koo, J. Park et al., "Prevention of radiocontrast medium-induced nephropathy using short-term high-dose simvastatin in patients with renal insufficiency undergoing coronary angiography (PROMISS) trial-a randomized controlled study," American Heart Journal, vol. 155, no. 3, pp. 499e1-499-e8, 2008.

[10] S. H. Jo, J. Y. Hahn, S. Y. Lee et al., "High-dose atorvastatin for preventing contrast-induced nephropathy in primary percutaneous coronary intervention," Journal of Cardiovascular Medicine (Hagerstown), 2014.

[11] A. Kaya, M. Kurt, I. H. Tanboğa et al., "Rosuvastatin versus atorvastatin to prevent contrast induced nephropathy in patients undergoing primary percutaneous coronary intervention (ROSA-cIN trial)," Acta Cardiologica, vol. 68, no. 5, pp. 489-494, 2013. 
[12] M. Leoncini, A. Toso, M. Maioli, F. Tropeano, S. Villani, and F. Bellandi, "Early high-dose rosuvastatin for contrast-induced nephropathy prevention in acute coronary syndrome: results from the PRATO-ACS study (protective effect of rosuvastatin and antiplatelet therapy on contrast-induced acute kidney injury and myocardial damage in patients with acute coronary syndrome)," Journal of the American College of Cardiology, vol. 63, no. 1, pp. 71-79, 2014.

[13] W. Li, X. Fu, Y. Wang et al., "Beneficial effects of high-dose atorvastatin pretreatment on renal function in patients with acute ST-segment elevation myocardial infarction undergoing emergency percutaneous coronary intervention," Cardiology, vol. 122, no. 3, pp. 195-202, 2012.

[14] H. Li, X. Li, H. Ma et al., "Atorvastatin combining with probucol: a new way to reduce serum uric acid level during perioperative period of interventional procedure," Scientific World Journal, vol. 2014, Article ID 565367, 6 pages, 2014.

[15] H. Özhan, I. Erden, S. Ordu et al., "Efficacy of short-term highdose atorvastatin for prevention of contrast-induced nephropathy in patients undergoing coronary angiography," Angiology, vol. 61, no. 7, pp. 711-714, 2010.

[16] G. Patti, E. Ricottini, A. Nusca et al., "Short-term, highdose Atorvastatin pretreatment to prevent contrast-induced nephropathy in patients with acute coronary syndromes undergoing percutaneous coronary intervention (from the ARMYDA-CIN [atorvastatin for reduction of myocardial damage during angioplasty-contrast-induced nephropathy] trial," American Journal of Cardiology, vol. 108, no. 1, pp. 1-7, 2011.

[17] C. Quintavalle, D. Fiore, F. de Micco et al., "Impact of a high loading dose of atorvastatin on contrast-induced acute kidney injury," Circulation, vol. 126, no. 25, pp. 3008-3016, 2012.

[18] A. Toso, M. Maioli, M. Leoncini et al., "Usefulness of Atorvastatin $(80 \mathrm{mg})$ in prevention of contrast-induced nephropathy in patients with chronic renal disease," The American Journal of Cardiology, vol. 105, no. 3, pp. 288-292, 2010.

[19] J. Xinwei, F. Xianghua, Z. Jing et al., "Comparison of usefulness of simvastatin $20 \mathrm{mg}$ versus $80 \mathrm{mg}$ in preventing contrastinduced nephropathy in patients with acute coronary syndrome undergoing percutaneous coronary intervention," The American Journal of Cardiology, vol. 104, no. 4, pp. 519-524, 2009.

[20] X. Zhou, Y. Jin, Q. Wang, R. Min, and X. Zhang, "Efficacy of high dose atorvastatin on preventing contrast induced nephropathy in patients underwent coronary angiography," Zhonghua Xin Xue Guan Bing Za Zhi, vol. 37, no. 5, pp. 394-396, 2009.

[21] T. Greco, G. Landoni, G. Biondi-Zoccai et al., "A Bayesian network meta-analysis for binary outcome: how to do it," Statistical Methods in Medical Research, 2013.

[22] F. D’Ascenzo and G. Biondi-Zoccai, "Network meta-analyses: the "White Whale" for cardiovascular specialists," Journal of Cardiothoracic and Vascular Anesthesia, vol. 28, no. 1, pp. 169173, 2013.

[23] G. Biondi-Zoccai, Network Meta-Analysis: Evidence Synthesis with Mixed Treatment Comparison, Nova Science Publishers, Hauppauge, NY, USA, 2014.

[24] G. G. L. Biondi-Zoccai, P. Agostoni, A. Abbate, L. Testa, F. Burzotta, and K. A. Robinson, "A simple hint to improve Robinson and Dickersin's highly sensitive PubMed search strategy for controlled clinical trials," International Journal of Epidemiology, vol. 34, no. 1, pp. 224-225, 2005.
[25] G. Biondi-Zoccai, M. Lotrionte, H. S. Thomsen et al., "Nephropathy after administration of iso-osmolar and lowosmolar contrast media: evidence from a network metaanalysis," International Journal of Cardiology, vol. 172, no. 2, pp. 375-80, 2014.

[26] C. Haller and I. Hizoh, "The cytotoxicity of iodinated radiocontrast agents on renal cells in vitro," Investigative Radiology, vol. 39, no. 3, pp. 149-154, 2004.

[27] A. Pisani, M. Sabbatini, E. Riccio et al., "Effect of a recombinant manganese superoxide dismutase on prevention of contrast-induced acute kidney injury," Clinical and Experimental Nephrology, vol. 181, no. 3, pp. 424-431, 2013.

[28] J. G. Webb, G. E. Pate, K. H. Humphries et al., "A randomized controlled trial of intravenous $\mathrm{N}$-acetylcysteine for the prevention of contrast-induced nephropathy after cardiac catheterization: lack of effect," American Heart Journal, vol. 148, no. 3, pp. 422-429, 2004.

[29] S. Khanal, N. Attallah, D. E. Smith et al., "Statin therapy reduces contrast-induced nephropathy: an analysis of contemporary percutaneous interventions," American Journal of Medicine, vol. 118, no. 8, pp. 843-849, 2005.

[30] R. E. Katholi, W. T. Woods Jr., G. J. Taylor et al., "Oxygen free radicals and contrast nephropathy," American Journal of Kidney Diseases, vol. 32, no. 1, pp. 64-71, 1998. 


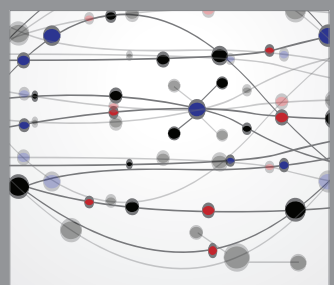

The Scientific World Journal
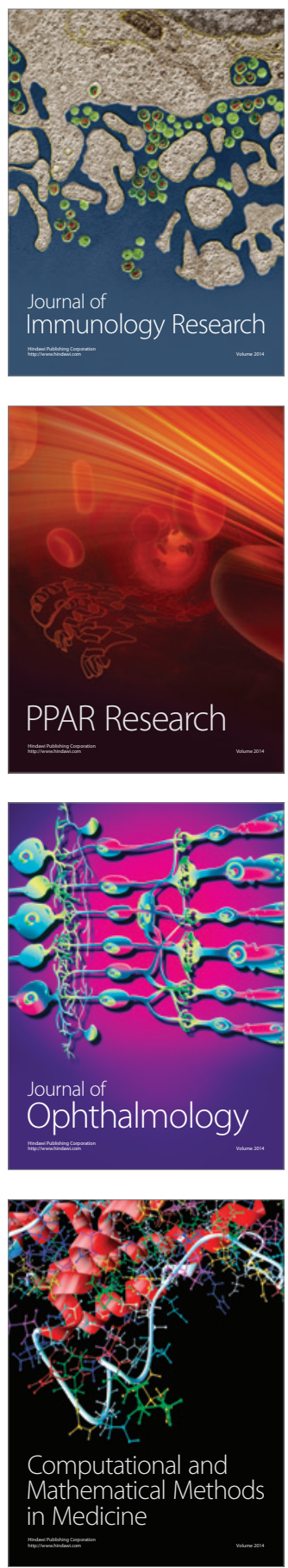

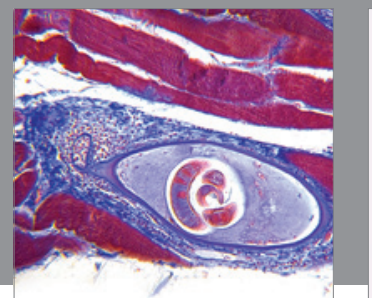

Gastroenterology

Research and Practice
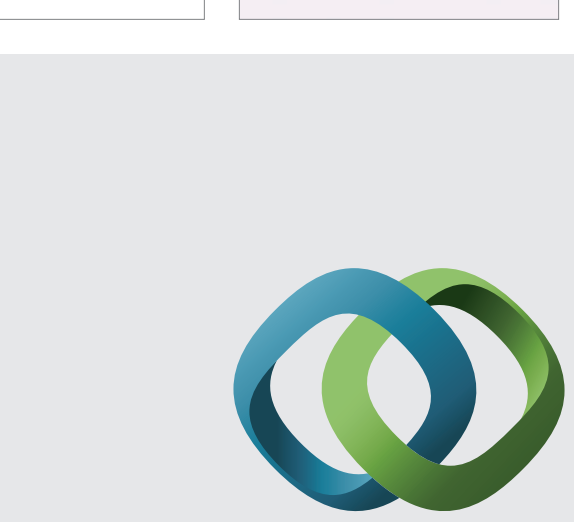

\section{Hindawi}

Submit your manuscripts at

http://www.hindawi.com
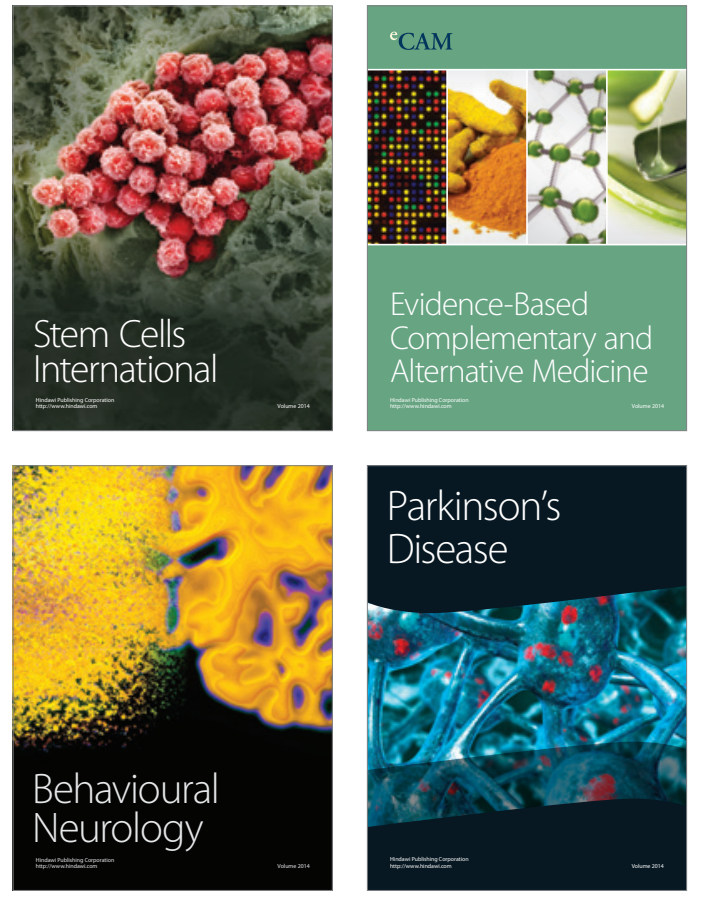
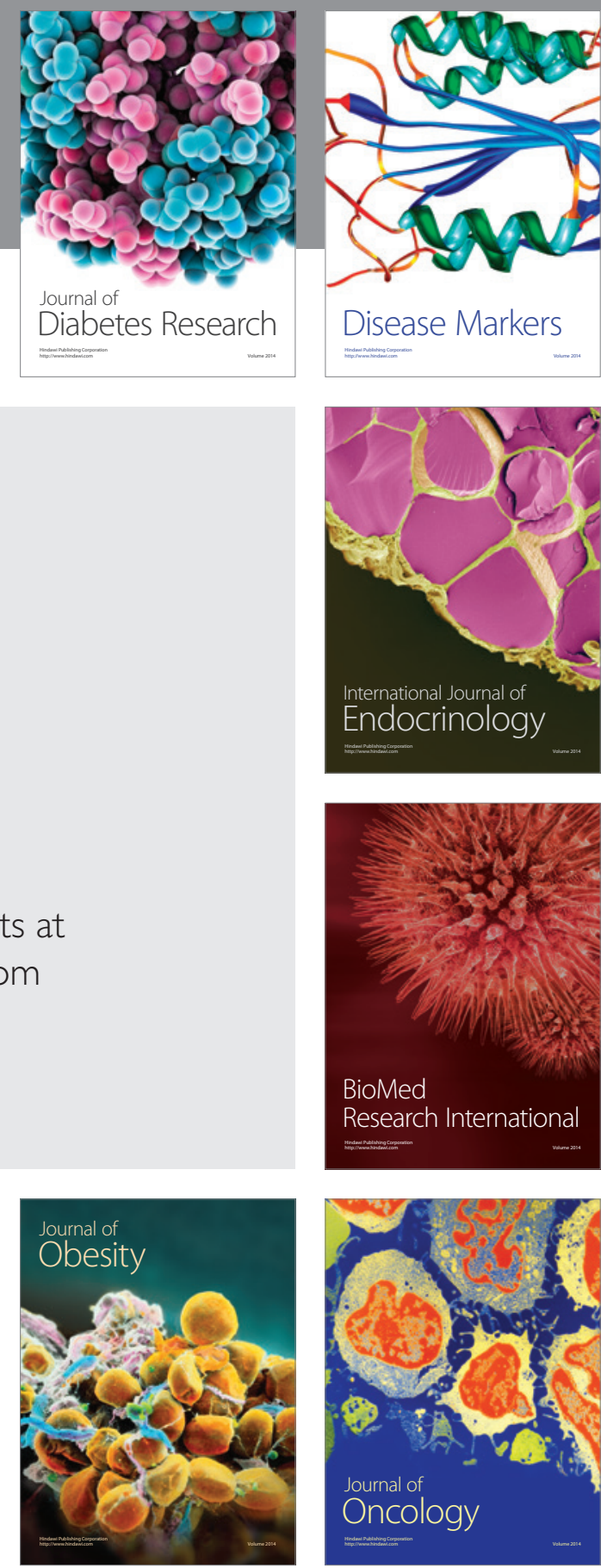

Disease Markers
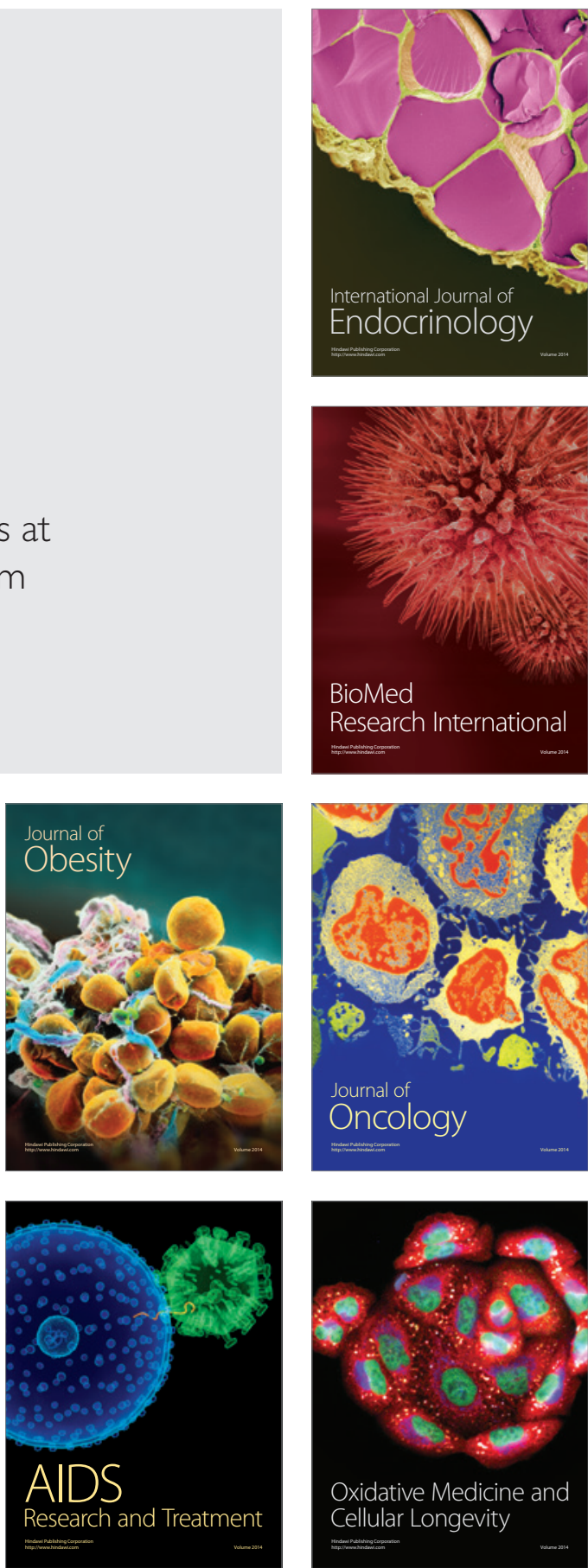